\title{
Simulation of a sponge iron production process
}

\author{
TOR ONSHUS $\dagger$
}

Keywords: sponge iron, shaft furnace, simulation, control.

A model for reduction of $\mathrm{FeO}$ with hydrogen in a countercurrent moving bed reactor is summarized. This model is a special case of a more complete model which also includes reduction of the higher oxides, hematite and magnetite, with a mixture of reducing gases, thus describing the production of direct-reduced iron from iron ores. Equations governing the heat and mass transfer between the gas and solid phase are not given here, but play an important role in the dynamic behaviour of the model.

The distributed parameter model is discretized, using Euler approximation for the time derivative and a backward difference to approximate the spatial derivatives.

Parameters applying to a pilot plant are used when simulating the model to study the approximate behaviour of the pilot plant. The model is then used to test a controller arrangement, using single loop PI-controllers. The dynamic responses that are shown indicate that this controller arrangement can be satisfactory.

\section{Direct reduction of iron}

Direct reduction is defined as any process in which metallic iron is produced by reduction of iron ore or other oxides, below the melting temperature of any of the materials involved. Because of the porous nature of the product, the name sponge iron is used.

The first iron produced was direct-reduced iron. Most of the oxygen chemically combined with iron was removed by direct reduction. Impurities were removed by reheating and mechanical working to make a solid piece of metal.

Today, most of the iron (pig iron) is produced in blast furnaces, based on a combination of direct reduction and melting. Approximately $1 / 30$ of the iron is produced in various direct reduction installations. The nearest is situated in Emden, West Germany, and utilizes gas from the North Sea as raw material for production of the reduction gas. The reaction is carried out in a shaft furnace. Descending solids (pelletized iron ores) are reduced by gas which is a mixture, mainly $\mathrm{CO}$ and $\mathrm{H}_{2}$, moving upwards.

Direct-reduced iron is today mainly used in three processes for further melting and treatment. In the electric arc furnace, it is used as a complete or partial substitute for various ferrous scrap. In the blast furnace it can be used to reduce the use of coke and increase the production. In basic oxygen furnaces, for refining of pig iron from the blast furnace, direct reduced iron can replace scrap by up to $70 \%$.

To study the gas solid interaction, a pilot plant (Fig. 1), $6 \mathrm{~m}$ high and $20 \mathrm{~cm}$ in inner diameter, is built. The composition of the reduction gas is arbitrarily mixed from $\mathrm{CO}$ and $\mathrm{H}_{2}$ and then split in two paths. One branch enters the shaft at the bottom and

Received 3 November 1982.

† University of Trondheim, The Norwegian Institute of Technology, Division of Engineering Cybernetics, N-7034 Trondheim-NTH, Norway. 


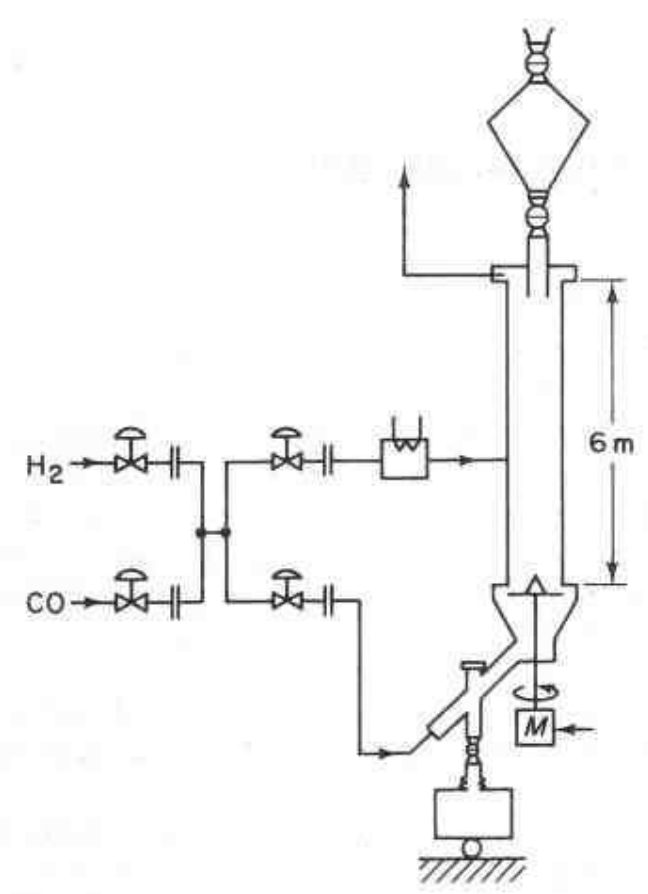

Figure 1. Pilot plant to study gas/solid reactions.

is used to cool the reduced pellets before they are discharged from the shaft. The rest of the gas is heated in a $20 \mathrm{~kW}$ carbon resistance heater before it enters the shaft approximately $2 \mathrm{~m}$ above the bottom. The maximum capacity is $30 \mathrm{~m}^{3} / \mathrm{h}$ for both $\mathrm{CO}$ and $\mathrm{H}_{2}$. Solid is fed from a gas sealed hopper on the top, and downwards velocity is controlled by rotation of a table feeder in the bottom. Because of the diameter of only $20 \mathrm{~cm}$, the shaft is equipped with $3 \times 4 \mathrm{~kW}$ electrical heating elements to reduce heat loss. The elements are distributed from the warm gas inlet and $3 \mathrm{~m}$ upwards. Each element is locally temperature controlled with external setpoint. The outlet gas at the top of the shaft is continuously analysed for $\mathrm{CO}, \mathrm{CO}_{2}, \mathrm{H}_{2} \mathrm{O}$ and $\mathrm{CH}_{4}$. It is also possible to extract gas from other parts of the shaft for analysis of the gas composition profile. On various locations along the shaft, thermocouples are inserted to measure temperature. All measurements (approx. 30) and control signals (approx. 10) are via 4-20 mA current loop, connected to a Camac process interface and a NORD-10 computer.

\section{Mathematical model}

To test various control strategies before a control system is implemented, a dynamic mathematical model is very useful. The control system can be changed and investigated by computer simulations without expensive and time-consuming experiments. (One experiment costs approximately $50000 \mathrm{Nkr}$ and takes one week.) Another aspect of modeling is the deeper insight and understanding of the physics of the process which the modeling gives.

The model is, like the experiments, conveniently divided into two interacting parts. The reaction rate and the heat transfer are mainly given from the local conditions around and inside each pellet, while the bulk transport is also driven by gradients over distances comparable to the shaft diameter. 
Here, a brief summary of the model for reduction of $\mathrm{FeO}$ (wystite) to Fe (iron) with $\mathrm{H}_{2}$ (hydrogen) is given. A detailed model of most of the reactions and reaction rates included in direct reduction is described in Onshus and Kolbeinsen 1981, 1981. The reaction studied here is:

$$
\mathrm{FeO}+\mathrm{H}_{2} \stackrel{R^{*}}{\longrightarrow} \mathrm{Fe}+\mathrm{H}_{2} \mathrm{O}
$$

By defining the degree of oxidation for solid,

$$
O x=\frac{C_{\mathrm{FeO}}}{C_{\mathrm{FeO}}+C_{\mathrm{Fe}}}
$$

the degree of oxidation for gas

$$
w=\frac{C_{\mathrm{H}_{2} \mathbf{O}}}{C_{\mathrm{H}_{2} \mathbf{O}}+C_{\mathbf{H}_{2}}}
$$

and using the principles of mass, molar and enthalpy conservation, one derives the following equations after some manipulation.

Gas composition

$$
C_{g}\left(\frac{d w}{d t}+v_{g} \frac{d w}{d z}\right)=R^{*}
$$

Solid composition

$$
C_{s}\left(\frac{d O x}{d t}+v_{s} \frac{d O x}{d z}\right)=-\frac{2}{3} R^{*}
$$

Gas temperature

$$
C_{g} C_{p g}\left(\frac{d T_{g}}{d t}+v_{g} \frac{d T_{g}}{d z}\right)+\left[h_{\mathrm{H}_{2} \mathrm{O}}\left(T_{g}\right)-h_{\mathrm{H}_{2} \mathrm{O}}\left(T_{s}\right)\right] R^{*}+h_{w}\left(T_{g}-T_{w}\right)+h_{v}\left(T_{\theta}-T_{s}\right)=0
$$

The wall temperature $T_{w}$ is computed under the assumption of stationary heat conduction through the shaft wall.

Solid temperature

$$
\begin{aligned}
C_{s} C_{p s}\left(\frac{d T_{s}}{d t}+v_{s} \frac{d T_{s}}{d z}\right)-\left[h_{\mathrm{H}_{2}}\left(T_{g}\right)-h_{\mathrm{H}_{2}}\left(T_{s}\right)\right] R^{*} & -h_{v}\left(T_{g}-T_{s}\right) \\
& +\left(h_{\mathrm{H}_{2} \mathrm{O}}-h_{\mathrm{H}_{2}}+h_{\mathrm{Fe}}-h_{\mathrm{FeO}}\right) R^{*}=0
\end{aligned}
$$

In the equations above, both solid and gas velocities are necessary. The solid velocity is given from the linear movement of solid. Gas velocity is more complex, as the behaviour according to the gas law has to be taken into account. In addition to gas temperature and mass velocity, the pressure drop in a packed bed has to be incorporated. Pressure drop in a packed bed of pellets is given by the Ergun equation (Bird 1960).

$$
\frac{d p}{d z}=-\frac{150}{d_{p}^{2}} \frac{(1-e)^{2}}{e^{3}} u v_{g}-\frac{1 \cdot 75}{d_{p}} \frac{(1-e)}{e^{3}} r v_{\theta}^{2}
$$

Steady state mass velocity is given by

$$
\frac{d G}{d z}=M_{o} R^{*}
$$


Mean gas velocity

$$
v_{\vartheta}=\frac{G}{C_{g} M_{g}}
$$

The eqns. (4)-(10) describe the interaction between gas and solid and are used to simulate partly what happens inside the pilot plant.

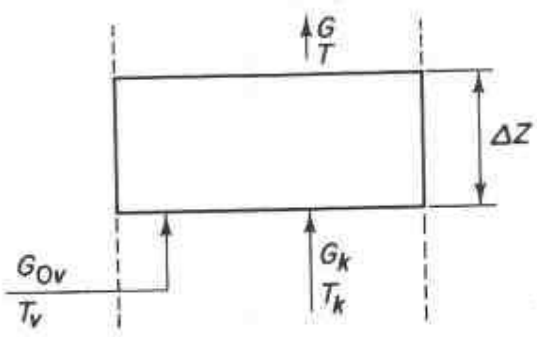

Figure 2. Mixing of gases with different composition and temperature at warm gas inlet.

Because of the warm gas inlet in the middle of the shaft, the mixing of gas at this point has to be computed separately. Using the physical balances in the mixing zone (Fig. 2), one gets the following equations for the mixing temperature, mass velocity, mean molar weight and gas composition.

$$
\begin{gathered}
f(T)=\frac{G}{M} h(T)-\frac{G_{k}}{M_{k}} h\left(T_{k}\right)-\frac{G_{0 v}}{M_{v}} h\left(T_{v}\right)+h_{w}\left(T-T_{w}\right) d z \\
+h_{v}\left(T-T_{s}\right) d z-\left(h_{\mathrm{H}_{2} \mathrm{O}}\left(T_{s}\right)-h_{\mathrm{H}_{2}}(T)\right) R^{*} d z=0 \\
G=G_{0 v}+G_{k}+M_{\mathrm{o}} R^{*} d z \\
M=\frac{G}{\frac{G_{0 v}}{M_{v}}+\frac{G_{k}}{M_{k}}} \\
w=\frac{M-M_{\mathrm{H}_{2}}}{M_{o}}
\end{gathered}
$$

Equation (11) is a non-linear equation in the temperature, because a non-linear approximation to heat capacity and thus enthalpy is used (Kabaschewski and Alcook 1979).

Before solving the equations, additional simplifications are made. Because of the great difference in heat capacity and flow velocities of gas and solids, one gets a stiff set of equations. To lessen the computational burden, some method of splitting of modi should be applied. Here the simplest method is used: By assuming that the gas dynamics are infinitely faster than that of the solids, the time-derivatives in eqns. (4) and (6) can be dropped.

\section{Numerical solution}

To solve the system of dynamic and stationary equations, the shaft (length $6 \mathrm{~m}$ ) is divided in 20 slices of $0.3 \mathrm{~m}$. Positive $z$-direction is defined upwards in the gas flow direction. Solids are then flowing in negative $z$-direction. 
Taking into account the physics of the convection process, the solid composition, eqn. (5), is solved using a simple first order approximation both in time and space.

$$
O x_{k}{ }^{+1}=O x_{k}{ }^{t}-\frac{v_{s} d t}{d z}\left(O x_{k}{ }^{2}-O x_{k+1}{ }^{t}\right)-\frac{2}{3} \frac{d t}{C_{s}} R^{*}
$$

where $k$ is space index and $t$ time index.

Solid temperature is computed in the same way, using Euler integration in time, and backward difference in space.

$$
\begin{aligned}
T_{s_{k}}{ }^{t+1}=T_{s_{k}}{ }^{t}-\frac{v_{s} d t}{d z}\left(T_{k}{ }^{t}-T_{k+1}{ }^{t}\right)+\frac{d t}{C_{s} C_{p s}}[ & h_{\mathrm{FeO}}\left(T_{s}\right)-h_{\mathrm{Fe}}\left(T_{s}\right)-h_{\mathrm{li}_{2} \mathrm{O}}\left(T_{s}\right) \\
& \left.+h_{\mathrm{li}_{2}}\left(T_{g}\right)\right] R^{*}+\frac{d t}{C_{s} C_{p s}} h_{v}\left(T_{g}-T_{s}\right)
\end{aligned}
$$

To get eqns. (15) and (16) started, the temperature $T_{s i}$ and the composition $O x_{i}$ at inlet on the top of the furnace are used.

The gas composition equantion is solved using.

$$
w_{k+1}{ }^{t+1}=w_{k}{ }^{t+1}+\left.\frac{d z}{v_{g} C_{g}} R^{*}\right|_{k+1}
$$

The same approximation for the space derivative as in eqn. (17) is also used to solve the gas temperature equation. But to get a more accurate answer, Newton iteration, eqns. (18)-(20), is applied in addition.

$$
T_{g k}{ }^{t+1}=T_{g k}{ }^{t+1}-\frac{f\left(T_{g k}{ }^{t+1}\right)}{\frac{d f\left(T_{g k}{ }^{t+1}\right)}{d T_{g k}{ }^{t+1}}}
$$

with

$$
\begin{aligned}
& f\left(T_{g k}{ }^{t+1}\right)=\left(C_{g} C_{p g} v_{g}\right)_{k} \frac{T_{g k}{ }^{t+1}-T_{g k-1}{ }^{t+1}}{d z}+\left(h_{v}\left(T_{g}-T_{s}\right)\right)_{k} \\
& +\left(h_{w}\left(T_{g}-T_{w}\right)\right)_{k}+\left(\left(h_{\mathrm{H}_{2} \mathrm{O}}\left(T_{g}\right)-h_{\mathrm{H}_{2} \mathrm{O}}\left(T_{s}\right)\right) R^{*}\right)_{k}
\end{aligned}
$$

and

$$
\frac{d}{d T_{g k}{ }^{t+1}}\left(f\left(T_{g k}{ }^{t+1}\right)\right)=\left(\frac{C_{g} C_{p g} v_{g}}{d z}+h_{v}+h_{w}+C_{p_{\mathrm{H}_{2} \mathrm{O}}} R^{*}\right)_{k}
$$

The equations concerning solid are solved from the top of the shaft, and downwards. The gas equations are, in accordance with gas motion, solved from the bottom and upwards. As initial values in the bottom, inlet composition of gas $w_{i}$ and temperature of cold gas $T_{g k}$ are used. To get the right temperature and composition above the mixing stage, eqns. (11) to (14) have to be solved. Newton iteration, eqn. (18), is applied together with the derivative given in eqn. (21), and the variables given by eqns. (11)-(14). When this iteration has converged, temperature $T$ and composition $w$ are used as initial values in eqns. (17)-(20) to give the solution for the upper part of the shaft.

$$
\frac{d f(T)}{d T}=C_{p g} \frac{G}{M}+C_{p_{\mathrm{H}_{2}}} R^{*} d z+\left(h_{v}+h_{w}\right) d z
$$

The eqns. (8)-(9), concerning gas velocity, are solved using the following schemes: Mass velocity of gas is integrated from the bottom to the warm gas inlet, with $G_{0 k}$, the 
inlet mass velocity of cooling gas, as initial value.

$$
G_{k+1}=G_{k}+d z M_{o} R^{*}{ }_{k}
$$

From the warm gas inlet and to the top, eqn. (22) is integrated using the value given by eqn. (12) as initial value.

The pressure equation is integrated from the top and down, applying the atmospheric pressure as initial value on the top.

$$
p_{k-1}=p_{k}+d z\left(-\frac{150}{d_{p}^{2}} \frac{(1-e)^{2}}{e^{3}} u v_{g}-\frac{1 \cdot 75}{d_{p}} \frac{(1-e)}{e^{3}} r v_{g}^{2}\right)_{k}
$$

The very simple solution method used here is by no means the best possible, it is selected to get an approximate answer as quick as possible. When all seven reactions and ten species appearing in direct reduction are included in the model, the nature of the numerical problem changes. The complete system then consists of ten continuity equations, together with two temperature equations like eqns. (6)-(7) and the pressure equation (8).

\section{Results from simulations}

Using numerical values given in Onshus and Kolbeinsen (1981), the equations are solved on a NORD-10 computer using Onshus (1977) giving the results shown in Figs. 3-10.

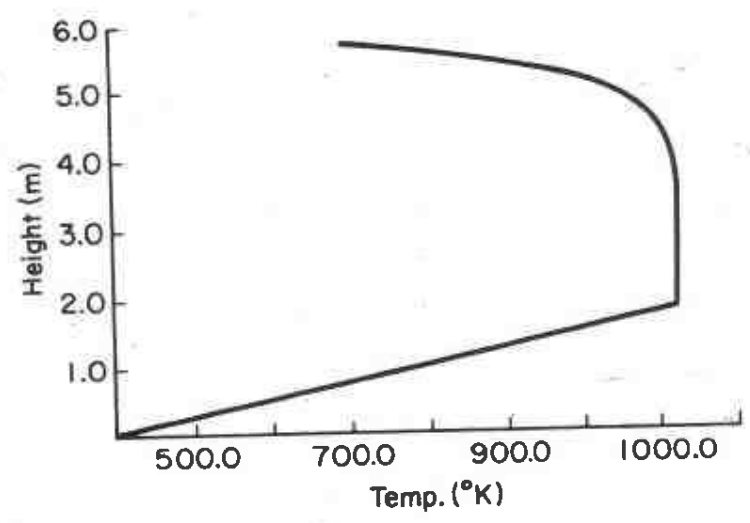

Figure 3. Profile of the solid temperature in the shaft.

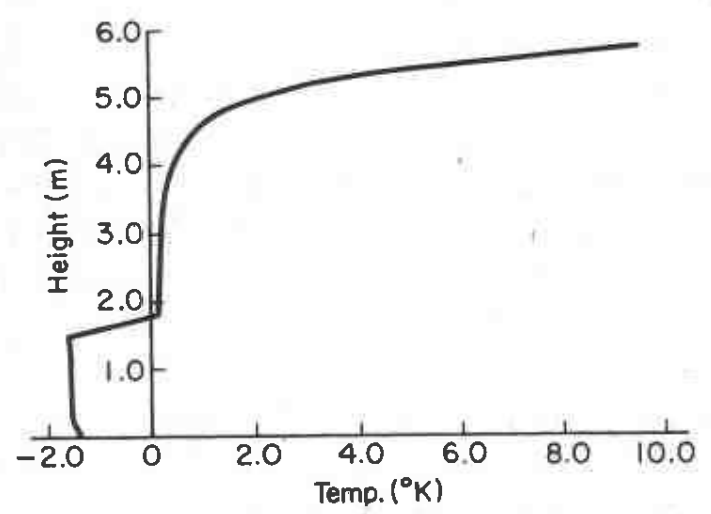

Figure 4. Profile of the temperature difference between gas and solid $T_{g}-T_{s}$. 


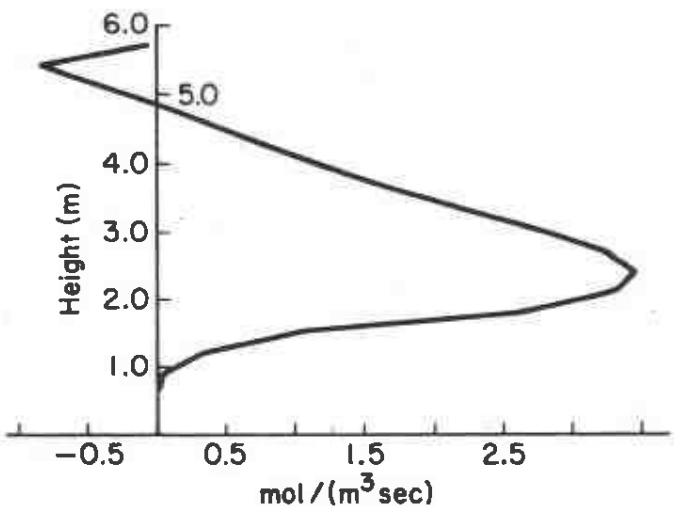

Figure 5. Profile of the reaction rate $R^{*}$ in shaft.

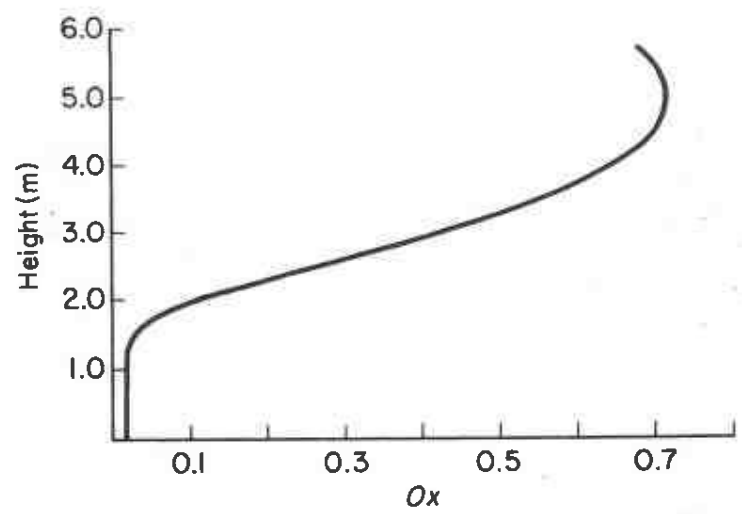

Figure 6. Profile of the degree of oxidation for solid $O x$.

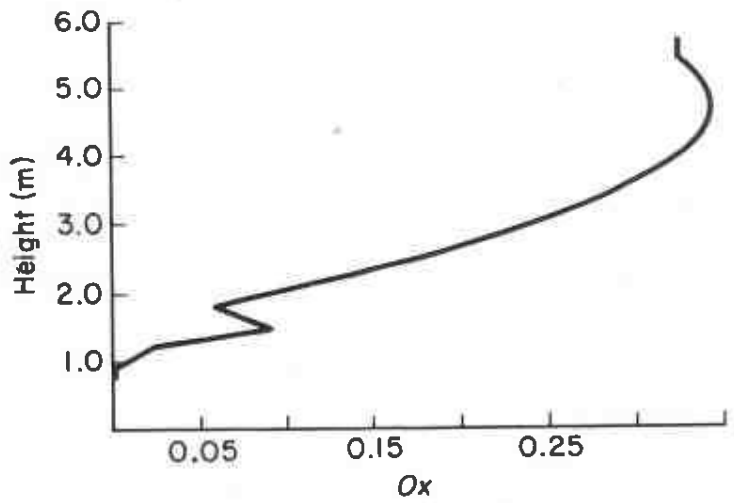

Figure 7. Profile of the degree of oxidation for gas $w$. 


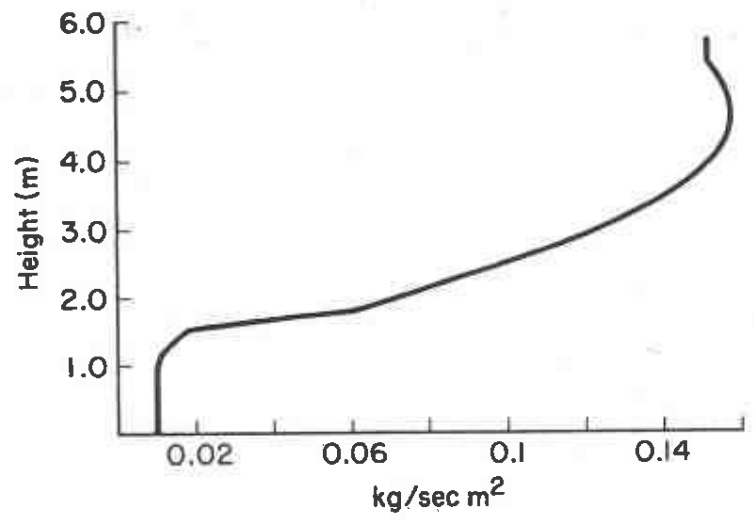

Figure 8. Profile of the mass velodity for gas $G$.

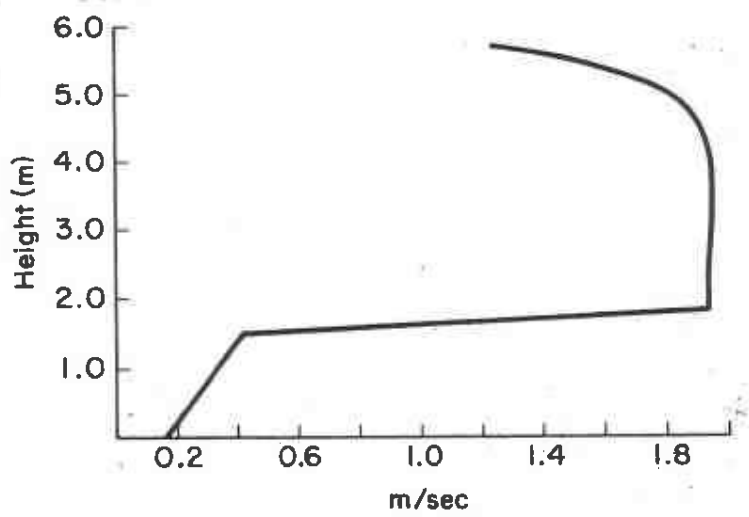

Figure 9. Profile of the gas velocity $V_{\theta}$.

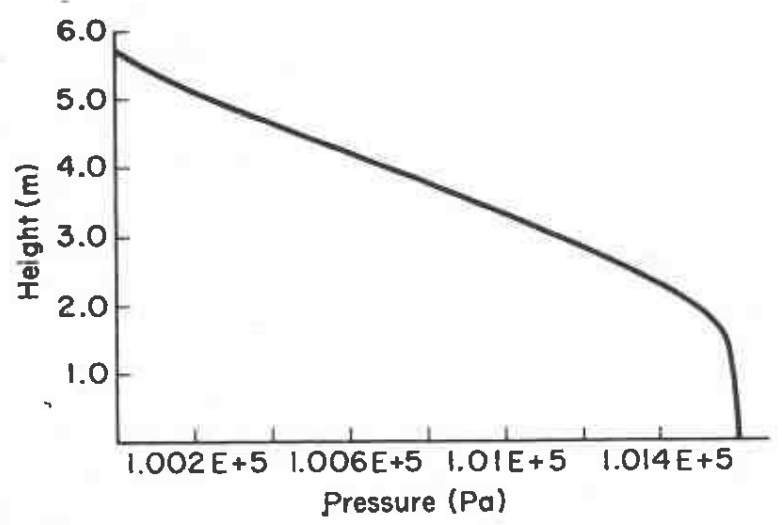

Figure 10. Profile of the gas pressure $p$. 
To test the dynamic behaviour of a closed loop system, a set of single loop PIcontrollers is used. The solid temperature from the simulations is used as measurements to the controllers.

The selection of measurements and connected control signal (see the Table) is done after studies of simulated responses to variations in control signals. Simulations are used specially at the warm gas input, where two independent control signal are available. These simulations show that the temperature of the warm gas decides the maximum value of the temperature-profile, while the mass velocity governs how fast the gas is cooled. This is because the product of temperature and mass velocity is proportional to the heat transferred into the shaft by the warm gas.

\begin{tabular}{ccccccc}
\hline $\begin{array}{c}\text { Measurement } \\
\text { at } z=\end{array}$ & $\begin{array}{c}\text { Steady } \\
\text { state } \\
\text { temp. }\end{array}$ & $\begin{array}{c}\text { Used to } \\
\text { control }\end{array}$ & $\begin{array}{c}\text { Steady } \\
\text { state } \\
\text { value }\end{array}$ & \multicolumn{2}{c}{$\begin{array}{c}\text { Controller } \\
K_{p}\end{array}$} & \multicolumn{1}{c}{$\begin{array}{c}\text { Reference } \\
\text { temperature } \\
t>0.1 \text { hour }\end{array}$} \\
\hline 0 & 405 & $G_{0 k}$ & 0.010 & -0.005 & 0 & 350 \\
$2 \cdot 4$ & 1126 & $T_{g v}$ & 1200 & 2.5 & 0.03 & 1150 \\
4.5 & 1117 & $G_{0 v}$ & 0.032 & 0.02 & 0 & 1150 \\
\hline
\end{tabular}

Parameters used to test temperature controller.

The following control algorithm is applied

$$
G_{0 k}=G_{0 k}{ }^{0}+K_{p}\left(T_{g}^{\mathrm{ref}}-T_{g}(z=0)\right)+K_{i} \int_{i=0}^{t}\left(T_{g}^{\mathrm{ref}}-T_{g}(z=0)\right) d t
$$

$G_{0 k}{ }^{0}$ is the steady state value of the control signal. A similar algorithm is used to control all three inputs $G_{0 k}, T_{g v}$ and $G_{0 v}$.

The references are, at $t=0 \cdot 1 \mathrm{~h}$, changed from the steady state values to those shown in the Table. The transient responses from these changes are shown in the Figs. 11-16.

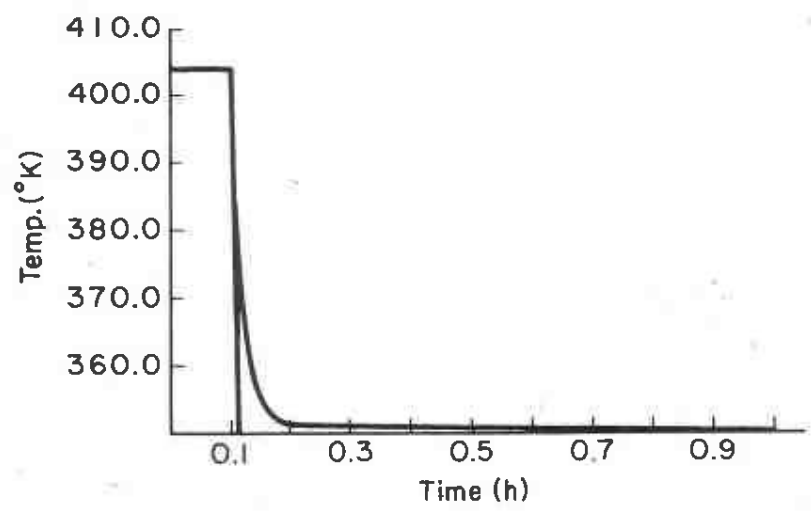

Figure 11. Time response of the solid temperature at the bottom of the shaft $(z=0)$ and the reference temperature. 


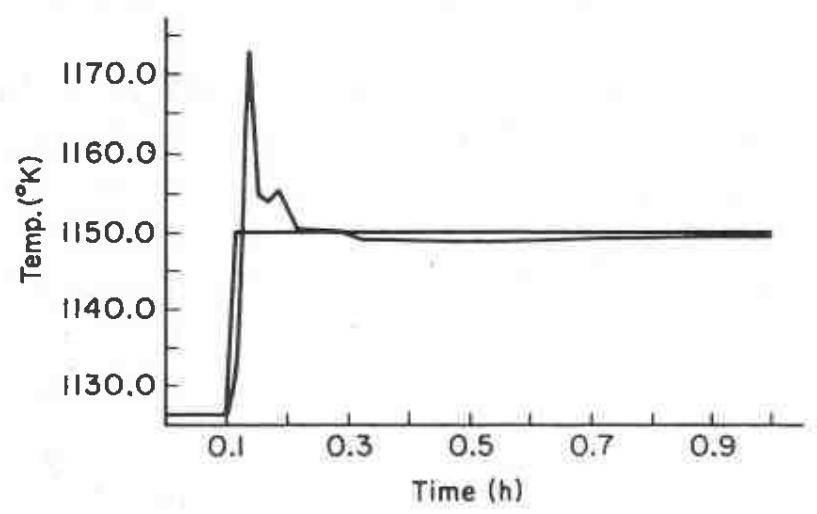

Figure 12. Time response of the solid temperature above warm gas inlet $(z=2.4 \mathrm{~m})$ and the reference temperature.

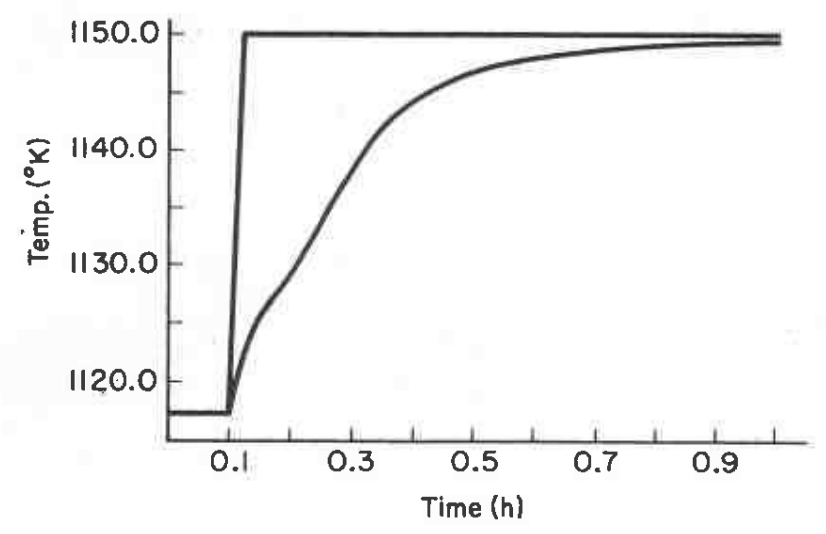

Figure 13. Time response of the solid temperature in the upper part of the shaft $(z=4.5 \mathrm{~m})$ and the reference temperature.

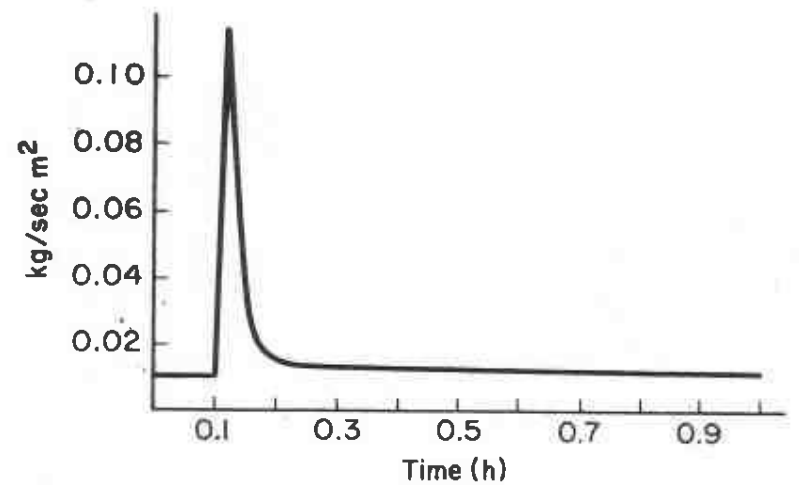

Figure 14. Time response of the controlled cold gas mass velocity. 


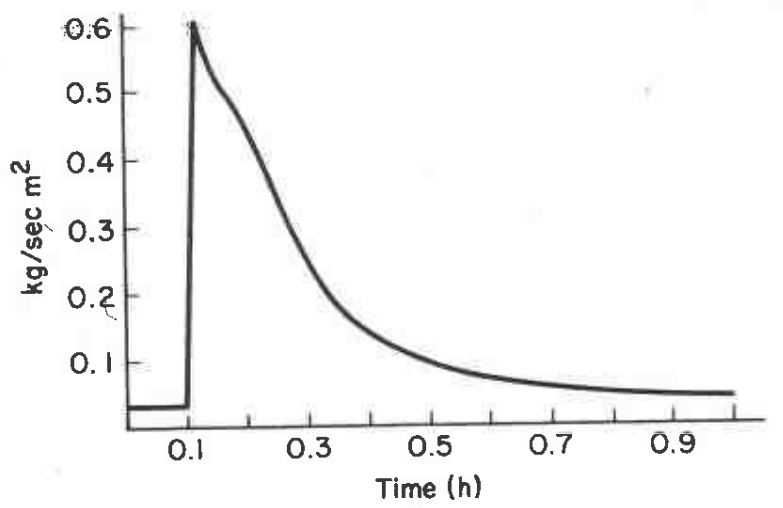

Figure 15. Time response of the controlled warm gas mass velocity.

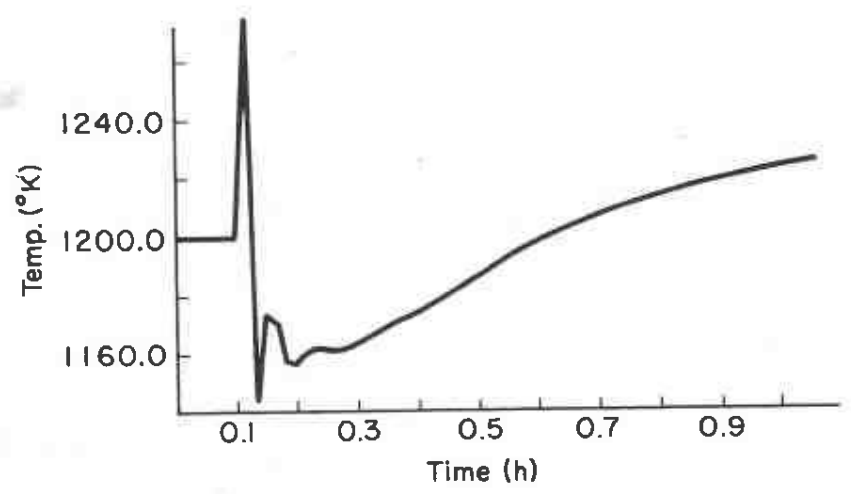

Figure 16. Time response of the controlled warm gas temperature.

\section{Concluding remarks}

The simulations show the use of a dynamic simulation model in the investigation of control problems. Of course, the parameters in the Table are not the first set tested but those which gave 'best' responses.

Although the control system works fairly well in simulations, the real test is when it is implemented on the pilot plant, maybe this autumn. Then measuring and modeling errors, in addition to the dynamics of control valves, etc. may cause trouble.

\section{Notation}

$C_{p g} \quad$ Heat capacity of gas

$\frac{\mathrm{J}}{\mathrm{mol}^{\circ} \mathrm{K}}$

$C_{p s} \quad$ Heat capacity of solid

$$
\frac{\mathbf{J}}{\mathrm{mol}{ }^{\circ} \mathrm{K}}
$$

$C_{g}$ Molar concentration of gas

$\frac{\mathrm{mol}}{\mathrm{m}^{3}}$

$C_{s}$ Molar concentration of solid

$$
\frac{\mathrm{mol}}{\mathrm{m}^{3}}
$$


$d_{p}$ Pellet diameter

m

$G$ Mass velocity of gas/area of shaft

$\frac{\mathrm{kg}}{\sec \mathrm{m}^{2}}$

$G_{0 k}$ Mass velocity of cold gas/area of shaft

$$
\frac{\mathrm{kg}}{\sec \mathrm{m}^{2}}
$$

$G_{0 \nu} \quad$ Mass velocity of war gas/area of shaft

$\frac{\mathrm{kg}}{\sec \mathrm{m}^{2}}$

$h_{\mathrm{H}_{2}}$ Enthalpy of hydrogen

$\frac{\mathrm{J}}{\mathrm{mol}}$

$h_{v}$ Heat transfer coefficient, gas-solid

$\frac{\mathrm{J}}{{ }^{0} \mathrm{~K} \mathrm{sec} \mathrm{m}^{3}}$

$h_{w}$ Heat transfer coefficient, gas-wall

$\frac{\mathrm{J}}{{ }^{\circ} \mathrm{K} \mathrm{sec} \mathrm{m}^{3}}$

$M_{g}$ Molar weight of gas

$\frac{\mathrm{kg}}{\mathrm{mol}}$

$M_{o} \quad$ Molar weight of oxygen

$\frac{\mathrm{kg}}{\mathrm{mol}}$

$M_{s} \quad$ Molar weight of solid

$\frac{\mathrm{kg}}{\mathrm{mol}}$

$O x$ Degree of oxidation $\left(O x=1\right.$ for $\left.\mathrm{Fe}_{2} \mathrm{O}_{3}\right)$

$O x_{l}$ Degree of oxidation at top of shaft

p Pressure

$\mathrm{Pa}=\frac{\mathrm{N}}{\mathrm{m}^{2}}$

$R$ Gas constant

$\frac{\mathrm{N} \mathrm{m}}{\mathrm{mol}{ }^{\circ} \mathrm{K}}$

$R^{*}$ Reaction rate

$\frac{\mathrm{mol}}{\mathrm{m}^{3} \mathrm{sec}}$

$r$ Specific density of gas

$\frac{\mathrm{kg}}{\mathrm{m}^{3}}$

$T_{g} \quad$ Gas temperature

${ }^{\circ} \mathrm{K}$

$T_{g k}$ Inlet gas temperature in bottom of shaft

${ }^{\circ} \mathrm{K}$

$T_{a v} \quad$ Inlet warm gas temperature

${ }^{\circ} \mathrm{K}$

$T_{s}$ Solid temperature

${ }^{\circ} \mathrm{K}$

$T_{s i}$ Solid temperature at top of shaft

${ }^{\circ} \mathrm{K}$

$u$ Viscosity of gas

$\frac{\mathrm{kg}}{\mathrm{m} \mathrm{sec}}$ 
$v_{g}$ Gas velocity

$z \quad$ Spatial coordinate (positive upwards)

$e$ Void fraction in packed bed

$w$ Degree of oxidation for gas

$w_{i}$ Degree of oxidation for gas at inlet

$$
\frac{\mathrm{m}}{\mathrm{sec}}
$$

m

\section{REFERENCES}

Onshus, T., and Kolbeinsen, L. (1981). STYRING AV SVAMPJERNPROSESSER. Matematisk modell for reduksjon av jernmalm med $\mathrm{CO} \mathrm{og} \mathrm{H}_{2}$ i motstrøms sjaktovn. STF48 A81058.

ONshus, T., and KOLBEINSEN, L. (1981). STYRING AV SVAMPJERNPROSESSER. Matematisk modell for reduksjon av $\mathrm{FeO}$ med $\mathrm{H}_{2}$ i motstrøms sjaktovn. STF34 A81010.

Bird, R. B., Stewart, W. E., LightFoot, E. N. (1960). Transport phenomena (John Wiley and Sons).

KubaschewsKı, O., and Alcocook, C. B. (1979). Metallurgical thermochemistry, 5th ed. (Pergamon Press, London).

Onshus, T. (1977). CYPROS del 1-Cybernetic Programs-STF48 F77075. 